\title{
Observability Analysis of Opportunistic Navigation with Pseudorange Measurements
}

\author{
Zaher M. Kassas*, and Todd E. Humphreys ${ }^{\dagger}$ \\ The University of Texas at Austin, Austin, TX, 78712, USA
}

\begin{abstract}
The observability analysis of an opportunistic navigation (OpNav) environment whose states may be partially known is considered. An OpNav environment can be thought of as a radio frequency signal landscape within which a receiver locates itself in space and time by extracting information from ambient signals of opportunity (SOPs). Available SOPs may have a fully-known, partially-known, or unknown characterization. In the present work, the receiver is assumed to draw only pseudorange-type measurements from the SOP signals. Separate observations are fused to produce an estimate of the receiver's position, velocity, and time (PVT). Since not all SOP states in the OpNav environment may be known a priori, the receiver must estimate the unknown SOP states of interest simultaneously with its own PVT. The observability analysis presented here first evaluates various linear and nonlinear observability tools and identifies those that can be appropriately applied to OpNav observability. Subsequently, the appropriate tools are invoked to establish the minimal conditions under which the environment is observable. It is shown that a planar OpNav environment consisting of a receiver and $n$ SOPs is observable if either the receiver's initial state is known or the receiver's initial position is known along with the initial state of one SOP. Simulation results are shown to agree with the theoretical observability conditions.
\end{abstract}

\section{Introduction}

Opportunistic navigation (OpNav) aims to extract positioning and timing information from ambient radio-frequency "signals of opportunity" (SOPs). OpNav radio receivers continuously search for opportune signals from which to draw navigation and timing information, employing on-the-fly signal characterization as necessary. Signals from discovered SOPs are downmixed and sampled coherently, yielding a tight coupling between signal streams that permits carrier-phase-level fusion of observables from the various streams within a single or distributed state estimator. ${ }^{1,2}$

In its most general form, OpNav treats all ambient radio signals as potential SOPs, from conventional global navigation satellite system (GNSS) signals to communications signals never intended for use as a timing or positioning source. Each signal's relative timing and frequency offsets, transmit location, and frequency stability, are estimated on-the-fly as necessary, with prior information about these quantities exploited when available. At this level of generality, the OpNav estimation problem is similar to the so-called simultaneous localization and mapping (SLAM) problem in robotics. ${ }^{3,4}$ Both imagine an actor which, starting with incomplete knowledge of its location and surroundings, simultaneously builds a map of its environment and locates itself within that map.

In traditional SLAM, the map that gets constructed as the actor (typically a robot) moves through the environment is composed of landmarks - walls, corners, posts, etc. - with associated positions. OpNav extends this concept to radio signals, with SOPs playing the role of landmarks. In contrast to a SLAM environmental map, the OpNav "signal landscape" is dynamic and more complex. For the simple case of pseudorange-only OpNav, where observables consist solely of signal time-of-arrival measurements, one must estimate, besides the three-dimensional position $\boldsymbol{x}_{s}$ and velocity $\dot{\boldsymbol{x}}_{s}$ of each SOP transmitter's antenna phase

*Graduate Research Assistant, Department of Electrical and Computer Engineering, zkassas@ieee.org

$\dagger$ Assistant Professor, Department of Aerospace Engineering and Engineering Mechanics, todd.humphreys@mail.utexas.edu 
center, each SOP's time offset $\delta t_{s}$ from a reference time base, rate of change of time offset $\dot{\delta} t_{s}$, and a small set of parameters that characterize the SOP's reference oscillator stability. Even more SOP parameters are required for an OpNav framework in which both pseudorange and carrier phase measurements are ingested into the estimator. ${ }^{1}$ Of course, in addition to the SOP parameters, the OpNav receiver's own position $\boldsymbol{x}_{r}$, velocity $\dot{\boldsymbol{x}}_{r}$, time offset $\delta t_{r}$, and time offset rate $\dot{\delta} t_{r}$ must be estimated.

The Global Positioning System (GPS) control segment routinely solves an instance of the OpNav problem: the location and timing offsets of a dozen or more GPS ground stations are simultaneously estimated with the orbital and clock parameters of the GPS satellites. ${ }^{5}$ Compared to the general OpNav problem, the GPS control segment's problem enjoys the constraints imposed by accurate prior estimates of site locations and satellite orbits. Moreover, estimation of clock states is aided by the presence of highly-stable atomic clocks in the satellites and at each ground station. In contrast, an OpNav receiver entering a new signal landscape may have less prior information to exploit and typically cannot assume atomic frequency references, either for itself or for the SOPs. The GPS control segment example also highlights the essentially collaborative nature of OpNav. Like the GPS ground stations, multiple OpNav receivers can share information to construct and continuously-refine a global signal landscape.

The large size of the OpNav estimation problem, which may involve hundreds of estimator states, naturally raises the question of state observability. As far as the authors are aware, no formal observability analysis has been done for opportunistic navigation as defined here. The primary goal of this paper is to theoretically determine the minimum conditions under which the pseudorange-only OpNav problem is observable.

A study of OpNav observability benefits from the OpNav-SLAM analogy. Although the question of observability was not addressed for more than a decade after SLAM was introduced, the recent SLAM literature has come around to considering fundamental properties of the SLAM problem, including observability. ${ }^{6-14}$ The effects of partial observability in planar SLAM with range and bearing measurements were first analyzed via linearization by Andrade-Cetto and Sanfeliu. ${ }^{6,7}$ These papers came to the counterintuitive conclusion that the two-dimensional planar wold-centric (absolute reference frame) SLAM problem is fully observable when the location of a single landmark is known a priori. With a fully nonlinear observability analysis, Lee et al. subsequently disproved this result and showed that at least two anchor landmarks with known positions are required for local weak observability. ${ }^{9}$ Later analysis of the SLAM problem's Fisher information matrix confirmed the result of the nonlinear analysis. ${ }^{10}$ However, an apparent discrepancy between linear and nonlinear SLAM observability analyses re-emerged in the work of Perera and Nettleton, ${ }^{11}$ where it was shown that a linear analysis based on piecewise constant system (PWCS) theory ${ }^{15}$ again predicted global planar-SLAM observability in the case of a single known anchor landmark, whereas a nonlinear analysis in the same paper indicated that two known anchor landmarks were required for local observability. The linear PWCS result appears flawed given that an observability test based on linearization should never predict observability in a case where a fully nonlinear test indicates lack of weak local observability. Given the confusion in the SLAM literature regarding the proper tools for observability analysis in SLAM-like problems, another goal of the current paper is to determine what observability tests are trustworthy in the context of opportunistic navigation.

Besides revealing the feasibility of state estimation, an observability analysis is also useful for designing more consistent estimators. For instance, Huang et al. exploit observability analysis to improve the consistency of Extended Kalman Filter (EKF)-based SLAM. ${ }^{13,14}$ Moreover, observability an analysis can guide maneuvers necessary to maintain accurate statistical estimates of the receiver and map. ${ }^{16}$

This paper makes two contributions. First, it compares the results of three different observability tests applied to two simple OpNav configurations to determine the appropriate tools for OpNav observability analysis. Second, it applies these tools to identify minimum conditions for pseudorange-only OpNav observability. For the sake of simplicity and physical intuition, similar to the SLAM observability studies, only the planar case will be considered in this paper. Extension of the planar analysis to three dimensions is anticipated to be straightforward.

The remainder of this paper is organized as follows. Section II reviews the various notions and tools 
necessary to analyze the observability of nonlinear systems, linear time-varying (LTV) systems, and linear PWCSs. Section III describes the OpNav environment model considered in this paper. Section IV analyzes various OpNav scenarios and establishes whether each scenario is observable. This leads to a set of minimum conditions necessary for complete OpNav observability. Section V presents simulation results on a number of scenarios, which confirm the conclusions of Section IV. Concluding remarks are given in Section VI.

\section{Theoretical Background}

Conceptually, observability of a dynamic system boils down to the question of solvability of the state from a linear or a nonlinear set of observations, where the state evolves according to a set of linear or nonlinear differential equations. In particular, observability is concerned with determining whether the state of the system can be consistently estimated from a set of observations over a finite period of time. For nonlinear systems, it is more appropriate to analyze the observability through nonlinear observability tools rather than linearizing the nonlinear system and applying linear observability tools. This is due to two reasons: $(i)$ nonlinear observability tools capture the nonlinearities of the dynamics and observations, and (ii) while the control inputs are never considered in the linear observability analysis, they are taken into account in the nonlinear observability analysis. It is instructive to define the various notions of observability and associated observability tests that will be invoked in the observability analysis of OpNav.

\section{A. Observability of Nonlinear Systems}

For the sake of clarity, various notions of nonlinear observability are defined in this subsection. ${ }^{17}$

Definition 1 Consider the continuous-time (CT) nonlinear dynamic system

$$
\Sigma_{\mathrm{NL}}:\left\{\begin{array}{l}
\dot{\boldsymbol{x}}(t)=\boldsymbol{f}[\boldsymbol{x}(t), \boldsymbol{u}(t)], \quad \boldsymbol{x}\left(t_{0}\right)=\boldsymbol{x}_{0} \\
\boldsymbol{y}(t)=\boldsymbol{h}[\boldsymbol{x}(t)],
\end{array}\right.
$$

with solution $\boldsymbol{x}(t)=\boldsymbol{g}\left(t, \boldsymbol{x}_{0}, \boldsymbol{u}\right)$, where $\boldsymbol{x} \in \mathbb{R}^{n}$ is the system state vector, $\boldsymbol{u} \in \mathbb{R}^{r}$ is the control input vector, $\boldsymbol{y} \in \mathbb{R}^{m}$ is the observation vector, and $\boldsymbol{x}_{0}$ is an arbitrary initial condition. Two states $\boldsymbol{x}_{1}$ and $\boldsymbol{x}_{2}$ are said to be indistinguishable if $\boldsymbol{h}\left[\boldsymbol{g}\left(t, \boldsymbol{x}_{1}, \boldsymbol{u}\right)\right]=\boldsymbol{h}\left[\boldsymbol{g}\left(t, \boldsymbol{x}_{2}, \boldsymbol{u}\right)\right]$, for all $t \geq 0$ and all $\boldsymbol{u}$. The set of all points indistinguishable from a particular state $\boldsymbol{x}$ is denoted as $\mathbb{I}(\boldsymbol{x})$.

Definition 2 The system $\Sigma_{\mathrm{NL}}$ is said to be observable at $\boldsymbol{x}_{0}$ if $\mathbb{I}\left(\boldsymbol{x}_{0}\right)=\left\{\boldsymbol{x}_{0}\right\}$. The system $\Sigma_{\mathrm{NL}}$ is said to be observable if $\mathbb{I}\left(\boldsymbol{x}_{0}\right)=\left\{\boldsymbol{x}_{0}\right\}$, for all $\boldsymbol{x}_{0} \in \mathbb{R}^{n}$.

Definition 3 Let $\mathfrak{N}$ be a subset (neighborhood) in the state-space $\mathbb{R}^{n}$ and $\boldsymbol{x}_{1}, \boldsymbol{x}_{2} \in \mathfrak{N}$. Two states $\boldsymbol{x}_{1}$ and $\boldsymbol{x}_{2}$ are said to be $\mathfrak{N}$-indistinguishable if every control $\boldsymbol{u}$, whose trajectories from $\boldsymbol{x}_{1}$ and $\boldsymbol{x}_{2}$ both lie in $\mathfrak{N}$, fails to distinguish between $\boldsymbol{x}_{1}$ and $\boldsymbol{x}_{2}$. The set of all $\mathfrak{N}$-indistinguishable states from a particular state $\boldsymbol{x}$ is denoted as $\mathbb{I}_{\mathfrak{N}}(\boldsymbol{x})$.

Definition 4 The system $\Sigma_{\mathrm{NL}}$ is said to be weakly observable at $\boldsymbol{x}_{0}$ if there exists a neighborhood $\mathfrak{N}$ such that $\mathbb{I}\left(\boldsymbol{x}_{0}\right) \cap \mathfrak{N}=\left\{\boldsymbol{x}_{0}\right\}$.

Definition 5 The system $\Sigma_{\mathrm{NL}}$ is said to be locally observable at $\boldsymbol{x}_{0}$ if $\mathbb{I}_{\mathfrak{N}}\left(\boldsymbol{x}_{0}\right)=\left\{\boldsymbol{x}_{0}\right\}$ for every open neighborhood $\mathfrak{N}$ of $\boldsymbol{x}_{0}$.

Definition 6 The system $\Sigma_{\mathrm{NL}}$ is said to be locally weakly observable at $\boldsymbol{x}_{0}$ if there exists an open neighborhood $\mathfrak{N}$ of $\boldsymbol{x}_{0}$ such that for every open neighborhood $\mathfrak{M}$ of $\boldsymbol{x}_{0}$ with $\mathfrak{M} \subset \mathfrak{N}, \mathbb{I}_{\mathfrak{M}}\left(\boldsymbol{x}_{0}\right)=\left\{\boldsymbol{x}_{0}\right\}$.

Intuitively, $\Sigma_{\mathrm{NL}}$ is locally weakly observable if $\boldsymbol{x}$ can be instantaneously distinguished from its neighbors. The various notions of observability are related to each other according to the following relationships

$$
\begin{array}{ccc}
\text { locally observable } & \Rightarrow \text { observable } \\
\Downarrow & \Downarrow \\
\text { locally weakly observable } & \Rightarrow \text { weakly observable. }
\end{array}
$$




\section{B. Observability of Linear Systems}

For linear time-invariant (LTI) systems, the four notions of nonlinear observability are equivalent. Observability of LTV systems is typically defined as follows. ${ }^{18}$

Definition 7 Consider the discrete-time (DT) linear LTV dynamic system

$$
\Sigma_{\mathrm{L}}:\left\{\begin{array}{l}
\boldsymbol{x}\left(t_{k+1}\right)=\mathbf{F}\left(t_{k}\right) \boldsymbol{x}\left(t_{k}\right)+\mathbf{B}\left(t_{k}\right) \boldsymbol{u}\left(t_{k}\right), \quad \boldsymbol{x}\left(t_{k_{0}}\right)=\boldsymbol{x}_{0}, k \in\left[t_{k_{0}}, t_{k_{f}}\right], \\
\boldsymbol{y}\left(t_{k}\right)=\mathbf{H}\left(t_{k}\right) \boldsymbol{x}\left(t_{k}\right),
\end{array}\right.
$$

where $\mathbf{F} \in \mathbb{R}^{n \times n}, \mathbf{B} \in \mathbb{R}^{n \times r}$, and $\mathbf{H} \in \mathbb{R}^{m \times n}$. The LTV system $\Sigma_{\mathrm{L}}$ is said to be observable in a time interval $\left[t_{k_{0}}, t_{k_{f}}\right]$, if the initial state $\boldsymbol{x}_{0}$ is uniquely determined by the zero-input response $\boldsymbol{y}\left(t_{k}\right)$ for $t_{k} \in\left[t_{k_{0}}, t_{k_{f-1}}\right]$. If this property holds regardless of the initial time $t_{k_{0}}$ or the initial state $\boldsymbol{x}_{0}$, the system is said to be completely observable.

\section{Nonlinear Observability Test}

For nonlinear systems, establishing global system properties, such as observability, is typically difficult to achieve. Hence, local properties are typically sought. A simple algebraic test exists for establishing local weak observability of a specific form of the nonlinear system $\Sigma_{\mathrm{NL}}$ in (1), known as the control affine form, ${ }^{19}$ given by

$$
\Sigma_{\mathrm{NL}, \mathrm{a}}:\left\{\begin{array}{l}
\dot{\boldsymbol{x}}(t)=\boldsymbol{f}_{0}[\boldsymbol{x}(t)]+\sum_{i=1}^{r} \boldsymbol{f}_{i}[\boldsymbol{x}(t)] u_{i}, \quad \boldsymbol{x}\left(t_{0}\right)=\boldsymbol{x}_{0} \\
\boldsymbol{y}(t)=\boldsymbol{h}[\boldsymbol{x}(t)] .
\end{array}\right.
$$

This test is based on the concept of Lie derivatives, which are defined next.

Definition 8 The first-order Lie derivative of a scalar function $h$ with respect to a vector-valued function $f$ is defined as

$$
\begin{aligned}
\mathfrak{L}_{\boldsymbol{f}}^{1} h(\boldsymbol{x}) & \triangleq \sum_{i=1}^{n} \frac{\partial h(\boldsymbol{x})}{\partial x_{i}} f_{i}(\boldsymbol{x}) \\
& =\nabla_{\boldsymbol{x}} h(\boldsymbol{x}) \cdot \boldsymbol{f}(\boldsymbol{x})
\end{aligned}
$$

The zeroth-order Lie derivative of any function is the function itself, i.e. $\mathfrak{L}_{\boldsymbol{f}}^{0} h(\boldsymbol{x})=h(\boldsymbol{x})$. The second-order Lie derivative can be computed recursively as

$$
\begin{aligned}
\mathfrak{L}_{\boldsymbol{f}}^{2} h(\boldsymbol{x}) & =\mathfrak{L}_{\boldsymbol{f}}\left[\mathfrak{L}_{\boldsymbol{f}}^{1} h(\boldsymbol{x})\right] \\
& =\left[\nabla_{\boldsymbol{x}} \mathfrak{L}_{\boldsymbol{f}}^{1} h(\boldsymbol{x})\right] \cdot \boldsymbol{f}(\boldsymbol{x}) .
\end{aligned}
$$

Higher-order Lie derivatives can be computed similarly.

Definition 9 Given the nonlinear system in control affine form $\Sigma_{\mathrm{NL}, \mathrm{a}}$. Define the matrix $\mathbf{G}$ as the matrix of zero-order through $(n-1)$-order of Lie derivatives

$$
\mathbf{G} \triangleq\left[\begin{array}{c}
\mathfrak{L}_{\boldsymbol{f}}^{0} h_{1}(\boldsymbol{x}) \\
\vdots \\
\mathfrak{L}_{\boldsymbol{f}}^{0} h_{m}(\boldsymbol{x}) \\
\hline \mathfrak{L}_{\boldsymbol{f}}^{1} h_{1}(\boldsymbol{x}) \\
\vdots \\
\frac{\mathfrak{L}_{\boldsymbol{f}}^{1} h_{m}(\boldsymbol{x})}{\vdots} \\
\hline \mathfrak{L}_{\boldsymbol{f}}^{n-1} h_{1}(\boldsymbol{x}) \\
\vdots \\
\mathfrak{L}_{\boldsymbol{f}}^{n-1} h_{m}(\boldsymbol{x})
\end{array}\right] .
$$


The so-called nonlinear observability matrix is defined as the gradient matrix of $\mathbf{G},{ }^{17}$ specifically

$$
\boldsymbol{O}_{\mathrm{NL}} \triangleq \nabla_{\boldsymbol{x}} \mathbf{G}=\left[\begin{array}{ccc}
\frac{\partial \mathfrak{S}_{f}^{0} h_{1}(\boldsymbol{x})}{\partial x_{1}} & \cdots & \frac{\partial \mathfrak{S}_{f}^{0} h_{1}(\boldsymbol{x})}{\partial x_{n}} \\
\vdots & \ddots & \vdots \\
\frac{\partial \mathfrak{L}_{f}^{0} h_{m}(\boldsymbol{x})}{\partial x_{1}} & \cdots & \frac{\partial \mathfrak{L}_{f}^{0} h_{m}(\boldsymbol{x})}{\partial x_{n}} \\
\hline \frac{\partial \mathfrak{S}_{f}^{1} h_{1}(\boldsymbol{x})}{\partial x_{1}} & \cdots & \frac{\partial \mathfrak{S}_{f}^{1} h_{1}(\boldsymbol{x})}{\partial x_{n}} \\
\vdots & \ddots & \vdots \\
\frac{\partial \mathfrak{L}_{f}^{1} h_{m}(\boldsymbol{x})}{\partial x_{1}} & \cdots & \frac{\partial \mathfrak{L}_{f}^{1} h_{m}(\boldsymbol{x})}{\partial x_{n}} \\
\hline \vdots & \ddots & \vdots \\
\frac{\partial \mathfrak{S}_{f}^{n-1} h_{1}(\boldsymbol{x})}{\partial x_{1}} & \cdots & \frac{\partial \mathfrak{S}_{f}^{n-1} h_{1}(\boldsymbol{x})}{\partial x_{n}} \\
\vdots & \ddots & \vdots \\
\frac{\partial \mathfrak{L}_{f}^{n-1} h_{m}(\boldsymbol{x})}{\partial x_{1}} & \cdots & \frac{\partial \mathfrak{L}_{f}^{n-1} h_{m}(\boldsymbol{x})}{\partial x_{n}}
\end{array}\right]
$$

The significance of the nonlinear observability matrix is that it can be employed to furnish necessary and sufficient conditions for local weak observability. ${ }^{17}$ In particular, if $\mathcal{O}_{\mathrm{NL}}$ is full-rank, then the system $\Sigma_{\mathrm{NL}, \mathrm{a}}$ is said to satisfy the observability rank condition.

Theorem 1 If the nonlinear system in control affine form $\Sigma_{\mathrm{NL}, \mathrm{a}}$ satisfies the observability rank condition, then the system is locally weakly observable.

Theorem 2 If a system $\Sigma_{\mathrm{NL}, \mathrm{a}}$ is locally weakly observable, then the observability rank condition is satisfied generically.

The term "generically" means that the observability matrix is full-rank everywhere, except possibly within a subset of the domain of $\boldsymbol{x} \cdot{ }^{20}$ Therefore, if the $\mathcal{O}_{\mathrm{NL}}$ is not of sufficient rank for all values of $\boldsymbol{x}$, the system is not locally weakly observable. ${ }^{21}$

\section{Linear Observability Tests}

Observability of LTV systems $\Sigma_{\mathrm{L}}$ is typically established through studying the rank of either the so-called observability Grammian or the observability matrix. The following theorem states a necessary and sufficient condition for observability of LTV systems through the $l$-step observability matrix. ${ }^{18}$

Theorem 3 The LTV system $\Sigma_{\mathrm{L}}$ is l-step observable if and only if the l-step observability matrix, defined as

$$
\mathcal{O}_{L}\left(t_{k}, t_{k+l}\right) \triangleq\left[\begin{array}{c}
\mathbf{H}\left(t_{k}\right) \\
\mathbf{H}\left(t_{k+1}\right) \mathbf{\Phi}\left(t_{k+1}, t_{k}\right) \\
\vdots \\
\mathbf{H}\left(t_{k+l-1}\right) \mathbf{\Phi}\left(t_{k+l-1}, t_{k}\right)
\end{array}\right]
$$

is full-rank, i.e. $\operatorname{rank}\left[\mathcal{O}_{L}\left(t_{k}, t_{k+l}\right)\right]=n$. The matrix $\boldsymbol{\Phi}$ is the DT transition matrix, defined as

$$
\mathbf{\Phi}\left(t_{k}, t_{j}\right) \triangleq \begin{cases}\mathbf{A}\left(t_{k-1}\right) \mathbf{A}\left(t_{k-2}\right) \cdots \mathbf{A}\left(t_{j}\right), & t_{k} \geq t_{j+1} \\ \mathbf{I}, & t_{k}=t_{j} .\end{cases}
$$

Another test to to establish observability of the LTV system $\Sigma_{\mathrm{L}}$ can be derived if the LTV system is piecewise constant. Observability of PWCSs has been analyzed for CT and DT systems. ${ }^{15}$ Observability of DT PWCSs is considered next.

Definition 10 An LTV system

$$
\Sigma_{\mathrm{L}, \mathrm{pwcs}}:\left\{\begin{array}{l}
\boldsymbol{x}\left(t_{k+1}\right)=\mathbf{F}_{j}\left(t_{k}\right) \boldsymbol{x}\left(t_{k}\right)+\mathbf{B}_{j}\left(t_{k}\right) \boldsymbol{u}\left(t_{k}\right), \quad \boldsymbol{x}\left(t_{0}\right)=\boldsymbol{x}_{0}, j=1, \ldots, r \\
\boldsymbol{y}\left(t_{k}\right)=\mathbf{H}_{j}\left(t_{k}\right) \boldsymbol{x}\left(t_{k}\right),
\end{array}\right.
$$

is said to be piecewise constant if for every time segment $j$, the matrices $\mathbf{F}_{j}, \mathbf{B}_{j}$, and $\mathbf{H}_{j}$ are constant, i.e. $\mathbf{F}_{j}\left(t_{k}\right)=\mathbf{F}_{j}, \mathbf{B}_{j}\left(t_{k}\right)=\mathbf{B}_{j}$, and $\mathbf{H}_{j}\left(t_{k}\right)=\mathbf{H}_{j}$. These matrices may vary from one segment to another. 
Definition 11 The instantaneous observability matrix of the PWCS $\Sigma_{\mathrm{L} \text {,pwcs }}$ in segment $j$ is defined as

$$
\mathcal{O}_{j}=\left[\begin{array}{c}
\mathbf{H}_{j} \\
\mathbf{H}_{j} \mathbf{F}_{j} \\
\mathbf{H}_{j} \mathbf{F}_{j}^{2} \\
\vdots \\
\mathbf{H}_{j} \mathbf{F}_{j}^{n-1}
\end{array}\right]
$$

Definition 12 The total observability matrix (TOM) of the PWCS $\Sigma_{\mathrm{L}, \mathrm{pwcs}}$ up to segment $r$ is defined as

$$
\mathcal{O}_{\mathrm{TOM}}(r)=\left[\begin{array}{c}
\mathcal{O}_{1} \\
\mathcal{O}_{2} \mathbf{F}_{1}^{n-1} \\
\mathcal{O}_{3} \mathbf{F}_{2}^{n-1} \mathbf{F}_{1}^{n-1} \\
\vdots \\
\mathcal{O}_{r} \mathbf{F}_{r-1}^{n-1} \mathbf{F}_{r-2}^{n-1} \cdots \mathbf{F}_{1}^{n-1}
\end{array}\right]
$$

Theorem 4 The DT PWCS system $\Sigma_{\mathrm{L}, \mathrm{pwcs}}$ is observable if and only if the TOM is full-rank, i.e. $\operatorname{rank}\left[\mathcal{O}_{\mathrm{TOM}}(r)\right]=$ $n$.

\section{Model Description}

This section describes the dynamics and observation model of the OpNav environment. While the observability analysis tools discussed in Section II apply to any receiver dynamics model, a reasonably simple planar receiver dynamics model is assumed, which does not obscure our physical intuition about the OpNav environment. More complicated receiver dynamics models will be investigated in future work.

\section{A. Dynamics Model}

The receiver's dynamics will be assumed to evolve according to the continuous white noise acceleration model, which is also known as the velocity random walk model. An object moving according to such dynamics in a generic coordinate $\xi$, has the dynamics

$$
\ddot{\xi}(t)=\tilde{w}_{\xi}(t),
$$

where $\tilde{w}_{\xi}(t)$ is a zero-mean white noise process with power spectral density $\tilde{q}_{\xi}$, i.e.

$$
\mathbb{E}\left[\tilde{w}_{\xi}(t)\right]=0, \quad \mathbb{E}\left[\tilde{w}_{\xi}(t) \tilde{w}_{\xi}(\tau)\right]=\tilde{q}_{\xi} \delta(t-\tau) .
$$

The receiver's clock errors will be modeled according to the two-state model, comprised of the clock bias $\delta t_{r}$ and clock drift $\dot{\delta} t_{r}$. The receiver's clock error states evolve according to

$$
\frac{d}{d t}\left[\begin{array}{c}
\delta t_{r}(t) \\
\dot{\delta} t_{r}(t)
\end{array}\right]=\left[\begin{array}{cc}
0 & 1 \\
0 & 0
\end{array}\right]\left[\begin{array}{c}
\delta t_{r}(t) \\
\dot{\delta} t_{r}(t)
\end{array}\right]+\left[\begin{array}{c}
\tilde{w}_{\delta t_{r}}(t) \\
\tilde{w}_{\delta t_{r}}(t)
\end{array}\right],
$$

where $\tilde{w}_{\delta t_{r}}$ and $\tilde{w}_{\dot{\delta} t_{r}}$ are zero-mean, mutually independent white noise processes with power spectra $S_{\delta t_{r}}$ and $S_{\dot{\delta} t_{r}}$, respectively. The power spectra $S_{\delta t_{r}}$ and $S_{\dot{\delta} t_{r}}$ are related to the power-law coefficients, $\left\{h_{\alpha}\right\}_{\alpha=-2}^{2}$, which characterize the power spectral density of the fractional frequency deviation of an oscillator from nominal frequency. ${ }^{22}$ It is common to approximate such relationships by considering only the frequency random walk coefficient $h_{-2}$ and the white frequency coefficient $h_{0}$, which lead to $S_{\delta t_{r}} \approx \frac{h_{0}}{2}$ and $S_{\dot{\delta} t_{r}} \approx 2 \pi^{2} h_{-2} .{ }^{23,24}$

The receiver's state vector will be defined by augmenting the receiver's planar position and velocity states with its clock error states to yield the state-space realization

$$
\dot{\boldsymbol{x}}_{r}(t)=\mathbf{A}_{r} \boldsymbol{x}_{r}(t)+\mathbf{D}_{r} \tilde{\boldsymbol{w}}_{r}(t),
$$


where

$$
\boldsymbol{x}_{r}=\left[\begin{array}{c}
\boldsymbol{r}_{r} \\
\dot{\boldsymbol{r}}_{r} \\
\delta t_{r} \\
\dot{\delta} t_{r}
\end{array}\right], \quad \tilde{\boldsymbol{w}}_{r}=\left[\begin{array}{c}
\tilde{w}_{x} \\
\tilde{w}_{y} \\
\tilde{w}_{\delta t_{r}} \\
\tilde{w}_{\dot{\delta} t_{r}}
\end{array}\right], \quad \mathbf{A}_{r}=\left[\begin{array}{cccccc}
0 & 0 & 1 & 0 & 0 & 0 \\
0 & 0 & 0 & 1 & 0 & 0 \\
0 & 0 & 0 & 0 & 0 & 0 \\
0 & 0 & 0 & 0 & 0 & 0 \\
0 & 0 & 0 & 0 & 0 & 1 \\
0 & 0 & 0 & 0 & 0 & 0
\end{array}\right], \quad \mathbf{D}_{r}=\left[\begin{array}{cccc}
0 & 0 & 0 & 0 \\
0 & 0 & 0 & 0 \\
1 & 0 & 0 & 0 \\
0 & 1 & 0 & 0 \\
0 & 0 & 1 & 0 \\
0 & 0 & 0 & 1
\end{array}\right],
$$

where $\boldsymbol{r}_{r} \triangleq\left[x_{r}, y_{r}\right]^{\top}$. The receiver's dynamics in (14) is discretized at a sampling period $T \triangleq t_{k+1}-t_{k}$ to yield the DT-equivalent system

$$
\boldsymbol{x}_{r}\left(t_{k+1}\right)=\mathbf{F}_{r} \boldsymbol{x}_{r}\left(t_{k}\right)+\boldsymbol{w}_{r}\left(t_{k}\right), \quad k=0,1,2, \ldots,
$$

where $\boldsymbol{w}_{r}$ is a DT zero-mean white noise vector with covariance $\mathbf{Q}_{r}$, and

$$
\mathbf{F}_{r}=\left[\begin{array}{cccccc}
1 & 0 & T & 0 & 0 & 0 \\
0 & 1 & 0 & T & 0 & 0 \\
0 & 0 & 1 & 0 & 0 & 0 \\
0 & 0 & 0 & 1 & 0 & 0 \\
0 & 0 & 0 & 0 & 1 & T \\
0 & 0 & 0 & 0 & 0 & 1
\end{array}\right], \quad \mathbf{Q}_{r}=\left[\begin{array}{ccccccc}
\tilde{q}_{x} \frac{T^{3}}{3} & 0 & \tilde{q}_{x} \frac{T^{2}}{2} & 0 & 0 & 0 \\
0 & \tilde{q}_{y} \frac{T^{3}}{3} & 0 & \tilde{q}_{y} \frac{T^{2}}{2} & 0 & 0 \\
\tilde{q}_{x} \frac{T^{2}}{2} & 0 & \tilde{q}_{x} T & 0 & 0 & 0 \\
0 & \tilde{q}_{y} \frac{T^{2}}{2} & 0 & \tilde{q}_{y} T & 0 & 0 \\
0 & 0 & 0 & 0 & S_{\delta t_{r}} T+S_{\dot{\delta} t_{r}} \frac{T^{3}}{3} & S_{\dot{\delta} t_{r}} \frac{T^{2}}{2} \\
0 & 0 & 0 & 0 & S_{\dot{\delta} t_{r}} \frac{T^{2}}{2} & S_{\dot{\delta} t_{r}}^{T}
\end{array}\right] .
$$

The SOP will be assumed to be spatially stationary and its state will consist of its planar position and clock error states. As in the receiver's case, each SOP's clock error states will be modeled according to the two-state model comprised of the SOP's clock bias, $\delta t_{s}$, and drift, $\dot{\delta} t_{s}$. Hence, the SOP's dynamics can be described by the LTI state-space model

$$
\dot{\boldsymbol{x}}_{s}(t)=\mathbf{A}_{s} \boldsymbol{x}_{s}(t)+\mathbf{D}_{s} \tilde{\boldsymbol{w}}_{s}(t),
$$

where

$$
\boldsymbol{x}_{s}=\left[\begin{array}{c}
\boldsymbol{r}_{s} \\
\delta t_{s} \\
\dot{\delta} t_{s}
\end{array}\right], \quad \tilde{\boldsymbol{w}}_{s}=\left[\begin{array}{c}
\tilde{w}_{\delta t_{s}} \\
\tilde{w}_{\dot{\delta} t_{s}}
\end{array}\right], \quad \mathbf{A}_{s}=\left[\begin{array}{cccc}
0 & 0 & 0 & 0 \\
0 & 0 & 0 & 0 \\
0 & 0 & 0 & 1 \\
0 & 0 & 0 & 0
\end{array}\right], \quad \mathbf{D}_{s}=\left[\begin{array}{cc}
0 & 0 \\
0 & 0 \\
1 & 0 \\
0 & 1
\end{array}\right],
$$

where $\boldsymbol{r}_{s} \triangleq\left[x_{s}, y_{s}\right]^{\top}$. Discretizing the SOP's dynamics (16) at a sampling interval $T$ yields the DT-equivalent model

$$
\boldsymbol{x}_{s}\left(t_{k+1}\right)=\mathbf{F}_{s} \boldsymbol{x}_{s}\left(t_{k}\right)+\boldsymbol{w}_{s}\left(t_{k}\right),
$$

where $\boldsymbol{w}_{s}$ is a DT zero-mean white noise vector with covariance $\mathbf{Q}_{s}$, and

$$
\mathbf{F}_{s}=\left[\begin{array}{cccc}
1 & 0 & 0 & 0 \\
0 & 1 & 0 & 0 \\
0 & 0 & 1 & T \\
0 & 0 & 0 & 1
\end{array}\right], \quad \mathbf{Q}_{s}=\left[\begin{array}{cccc}
0 & 0 & 0 & 0 \\
0 & 0 & 0 & 0 \\
0 & 0 & S_{\delta t_{s}} T+S_{\dot{\delta} t_{s}} \frac{T^{3}}{3} & S_{\delta t_{s}} \frac{T^{2}}{2} \\
0 & 0 & S_{\dot{\delta} t_{s}} \frac{T^{2}}{2} & S_{\dot{\delta t_{s}}} T
\end{array}\right]
$$

Defining the augmented state as $\boldsymbol{x} \triangleq\left[\boldsymbol{x}_{r}, \boldsymbol{x}_{s}\right]^{\top}$ and the augmented process noise vector as $\boldsymbol{w} \triangleq\left[\boldsymbol{w}_{r}, \boldsymbol{w}_{s}\right]^{\top}$ yields the system dynamics

$$
\boldsymbol{x}\left(t_{k+1}\right)=\mathbf{F} \boldsymbol{x}\left(t_{k}\right)+\boldsymbol{w}\left(t_{k}\right)
$$

where $\mathbf{F}=\operatorname{diag}\left[\mathbf{F}_{r}, \mathbf{F}_{s}\right]$ and the covariance of $\boldsymbol{w}$ is $\mathbf{Q}=\operatorname{diag}\left[\mathbf{Q}_{r}, \mathbf{Q}_{s}\right]$. While the model defined in (18) considered only one SOP, the model can be readily extended to multiple SOPs by augmenting their corresponding states and dynamics. 


\section{B. Observation Model}

To properly model the pseudorange observations, we must consider three different time systems to keep track of time. The first is true time, $t$, which is also commonly referred to as GPS system time. The second time system is that of the receiver's clock, $t_{r}$. The third time system is that of the SOP's clock, $t_{s}$. The three time systems are related to each other according to

$$
\begin{aligned}
& t=t_{r}-\delta t_{r}(t) \\
& t=t_{s}-\delta t_{s}(t),
\end{aligned}
$$

where $\delta t_{r}(t)$ and $\delta t_{s}(t)$ measure the amount by which the receiver and SOP clocks are different from true time, respectively.

The pseudorange observation made by the receiver on a particular SOP is made in the receiver time and is modeled according to

$$
\rho\left(t_{r}\right)=\left\|\boldsymbol{r}_{r}\left[t_{r}-\delta t_{r}\left(t_{r}\right)\right]-\boldsymbol{r}_{s}\left[t_{r}-\delta t_{r}\left(t_{r}\right)-\delta t_{\mathrm{TOF}}\right]\right\|_{2}+c .\left\{\delta t_{r}\left(t_{r}\right)-\delta t_{s}\left[t_{r}-\delta t_{r}\left(t_{r}\right)-\delta t_{\mathrm{TOF}}\right]\right\}+\tilde{v}_{\rho}\left(t_{r}\right),
$$

where $c$ is the speed of light, $\delta t_{\mathrm{TOF}}$ is the time-of-flight of the signal from the SOP to the receiver, and $\tilde{v}_{\rho}$ is the error in the pseudorange measurement due to unmodeled effects, modeling errors, and measurement errors, which is modeled as a zero-mean white Gaussian noise process with power spectral density $\tilde{r}^{25}$ The first term in (21) is the true range between the receiver's position and the SOP's position at time-of-transmission of the signal, while the second term arises due to the non-ideal nature of the receiver and SOP clocks. The observation model in the form of (21) is not appropriate for our observability analysis as it suffers from two shortcomings: $(i)$ it is in a time system that is different than the one considered in deriving the system dynamics, and (ii) the observation model is a nonlinear function of the delayed system states. The first shortcoming can be dealt with by converting the observation model to true time. The second problem is commonly referred to as the output delay problem, in which the observations (outputs) are a delayed-version, deterministic or otherwise, of the system state. A common approach to deal with this problem entails discretizaion and state-augmentation. ${ }^{26}$ For simplicity, and in order not to introduce additional states in our model, proper approximations will be invoked to deal with the second shortcoming.

Converting the pseudorange model in (21) to true time, assuming the receiver's position to be approximately stationary within a time interval of $\delta t_{r}(t)$, and invoking the fact that the SOP's position $\boldsymbol{r}_{s}$ is stationary yields

$$
\rho(t) \approx\left\|\boldsymbol{r}_{r}(t)-\boldsymbol{r}_{s}(t)\right\|_{2}+c \cdot\left\{\delta t_{r}(t)-\delta t_{s}\left[t-\delta t_{r}(t)-\delta t_{\mathrm{TOF}}\right]\right\}+\tilde{v}_{\rho}(t) .
$$

Next, it is argued that $\delta t_{s}\left[t-\delta t_{r}(t)-\delta t_{\mathrm{TOF}}\right] \approx \delta t_{s}(t)$. The validity of this argument depends on the relative magnitudes of $\delta t_{\mathrm{TOF}}$ and how "wildly" the SOP and receiver clocks wander. For ground-based SOPs that are as far as $1 \mathrm{~km}$ away, the time-of-flight is of the order of $\delta t_{\mathrm{TOF}} \sim 3.33 \mu \mathrm{s}$. It is reasonable to assume the SOP clock bias $\delta t_{s}$ to have an approximately constant value around such $\delta t_{\mathrm{TOF}}$. Therefore, the pseudorange observation model can now be expressed as a nonlinear function of the state, namely

$$
z(t)=\rho(t) \triangleq h[\boldsymbol{x}(t)]+\tilde{v}_{\rho}(t) \approx\left\|\boldsymbol{r}_{r}(t)-\boldsymbol{r}_{s}(t)\right\|_{2}+c \cdot\left\{\delta t_{r}(t)-\delta t_{s}(t)\right\}+\tilde{v}_{\rho}(t) .
$$

Discretizing the observation equation at a sampling interval $T$ yields the DT-equivalent observation model

$$
z\left(t_{k}\right)=y\left(t_{k}\right)+v_{\rho}\left(t_{k}\right)=\left\|\boldsymbol{r}_{r}\left(t_{k}\right)-\boldsymbol{r}_{s}\left(t_{k}\right)\right\|_{2}+c .\left\{\delta t_{r}\left(t_{k}\right)-\delta t_{s}\left(t_{k}\right)\right\}+v_{\rho}\left(t_{k}\right),
$$

where $v_{\rho}$ is a DT zero-mean, white Gaussian process with covariance $r=\tilde{r} / T$.

\section{Observability Analysis with Pseudorange Observations}

This section first evaluates the three observability tests discussed in Section II by considering a simplified model, which we know how to analyze by physical intuition. Even though we may deduce a priori the observability conditions for this model, applying the nonlinear and linear observability tools will assess which tools are appropriate for analyzing various more complex OpNav scenarios. 


\section{A. Simple Scenario}

Consider the case of an environment with one unknown receiver and one fully-known anchor SOP, i.e. an SOP with known initial state. Moreover, assume that the receiver and SOP clocks are perfect. In this case, the state vector will consist of the receiver's position and velocity and the SOP's position, namely $\boldsymbol{x}=\left[x_{r}, y_{r}, \dot{x}_{r}, \dot{y}_{r}, x_{s, a}, y_{s, a}\right]^{\top}$. The observation in this case are the true range between the receiver and the SOP. Hence, the observation model is given by $\boldsymbol{y}(t)=\left[x_{s, a}(t), y_{s, a}(t),\left\|\boldsymbol{r}_{r}(t)-\boldsymbol{r}_{s}(t)\right\|\right]^{\top}$, where the two fictitious observations $x_{s, a}$ and $y_{s, a}$ were augmented to the observation vector to indicate knowledge of the position of the anchor SOP.

First, the nonlinear observability analysis is considered. Performing such analysis results in a nonlinear observability matrix $\mathcal{O}_{\mathrm{NL}}$ whose rank is 5 . Since $\mathcal{O}_{\mathrm{NL}}$ is rank-deficient, then by Theorem 2 we conclude that this system is unobservable. Even though the notion of "unobservable subspace" cannot be strictly defined for this system, by examining the physical interpretation of the basis of $\mathcal{O}_{\mathrm{NL}}^{\perp}$, i.e. the basis of the unobservable subspace, we will gain useful information. It turns out that a basis for the subspace $\mathcal{O}_{\mathrm{NL}}^{\perp}$ is given by

$$
\mathcal{O}_{\mathrm{NL}}^{\perp}=\operatorname{span}\left[\begin{array}{llllll}
\frac{-y_{r}+y_{s, a}}{\dot{x}_{r}} & \frac{x_{r}-x_{s, a}}{\dot{x}_{r}} & -\frac{\dot{y}_{r}}{\dot{x}_{r}} & 1 & 0 & 0
\end{array}\right]^{\top}=\operatorname{span}\left[\mathbf{n}_{1}\right] .
$$

The fact that the last two elements in the vector $\boldsymbol{n}_{1}$ are zeros imply that the states $x_{s, a}$ and $y_{s, a}$ are orthogonal to the unobservable subspace; hence, they are observable. This is of course case as such states are assumed to be known by construction.

Before employing the linear observability analysis tools, it is noted that while the dynamics are linear, the observations are nonlinear. Therefore, the model will be expressed in its linearized error form (also known as the indirect form), where the state vector $\Delta \boldsymbol{x}$ in this formulation contains the error states, which are defined as the difference between the true states and the nominal states, and the observation vector $\Delta \boldsymbol{z}$ is defined as the difference between the true observations and the nominal observations. The linearized error form of the system defined in (18) and (24) is given by

$$
\begin{aligned}
\Delta \boldsymbol{x}\left(t_{k+1}\right) & =\mathbf{F} \Delta \boldsymbol{x}\left(t_{k}\right) \\
\Delta \boldsymbol{y}\left(t_{k}\right) & =\mathbf{H}\left(t_{k}\right) \Delta \boldsymbol{x}\left(t_{k}\right),
\end{aligned}
$$

where $\mathbf{H}$ is the Jacobian matrix, evaluated at the nominal states.

Next, the LTV observability analysis through the $l$-step observability matrix $\mathcal{O}_{L}\left(t_{k}, t_{k+l}\right)$ is considered. Performing such analysis on the LTV in (25) yields a 1-step observability matrix $\mathcal{O}_{L}(0,1)$ whose rank is 3 , a 2-step observability matrix $\mathcal{O}_{L}(0,2)$ whose rank is 4 , and a 3 -step observability matrix $\mathcal{O}_{L}(0,3)$ whose rank is 5. Adding more time-steps does not improve the observability any further, and the $l$-step observability matrix will be always be rank-deficient by 1 , suggesting that the system is unobservable. In fact, the resulting null-space of $\mathcal{O}_{L}(0, l)$, for all $l \geq 3$, is given by

$$
\mathcal{N}\left[\mathcal{O}_{L}(0, l)\right]=\operatorname{span}\left[\begin{array}{llllll}
\frac{y_{s, a}-y_{r}}{\dot{x}_{r}} & \frac{-x_{s, a}+x_{r}}{\dot{x}_{r}} & -\frac{\dot{y}_{r}}{\dot{x}_{r}} & 1 & 0 & 0
\end{array}\right]^{\top} .
$$

It is worth noting that this null-space is identical to the subspace $\mathcal{O}_{\mathrm{NL}}^{\perp}$.

Third, the LTV observability analysis based on PWCS theory is considered. Performing such analysis on the LTV in (25) yields a TOM for the first time segment $\mathcal{O}_{\text {TOM }}(1)$ whose rank is 4 . The null-space for such matrix is given by

$$
\mathcal{N}\left[\mathcal{O}_{\mathrm{TOM}}(1)\right]=\operatorname{span}\left[\begin{array}{cccccc}
0 & 0 & -\frac{y_{r}-y_{s, a}+T \dot{y}_{r}}{x_{r}-x_{s, a}+T \dot{x}_{r}} & 1 & 0 & 0 \\
-\frac{y_{r}-y_{s, a}+T \dot{y}_{r}}{x_{r}-x_{s, a}+T \dot{x}_{r}} & 1 & 0 & 0 & 0 & 0
\end{array}\right]^{\top} .
$$

Adding a second time segment results in a full-rank $\mathcal{O}_{\mathrm{TOM}}(2)$; hence, according to Theorem 4 , the system is observable. Not only this conclusion contradicts the nonlinear observability analysis and the LTV observability analysis, but it also defies physical intuition. For the simple case under consideration, from 
physical intuition, we know that the OpNav environment is observable with at least two known SOP anchors. Performing the nonlinear observability and the LTV observability tests with 2 known anchor SOPs indeed result in full-rank $\mathcal{O}_{\mathrm{NL}}$ and $\mathcal{O}_{L}\left(t_{k}, t_{k+l}\right)$, respectively. Therefore, given the knowledge about the two anchor SOPs, the receiver's state maybe determined. Such determination will, however, be ambiguous, since there exists 2 indistinguishable initial conditions that result in the same observations.

As a concrete example, consider the scenario depicted in Figure 1. Here, $\mathrm{SOP}_{1}$ and $\mathrm{SOP}_{2}$ are of known positions and the receiver is moving along the dashed line with constant velocity. In this case, a receiver that starts from the initial state $\left(x_{r}(0), y_{r}(0)\right)$ and one that starts from the inital state $\left(x_{r}(0),-y_{r}(0)\right)$ will produce identical range measurements. Hence, these initial conditions are indistinguishable given the range measurement made by the receiver on both SOPs. In fact, it can be demonstrated that as long an estimator (e.g. EKF) is initialized with an initial estimate that lies in the same half-plane $(y>0$ or $y<0)$ as the true initial condition, the estimate will converge to the true state trajectory, while if the initial estimate is set to be in the opposite half-plane, it will converge to the opposite (incorrect) trajectory.

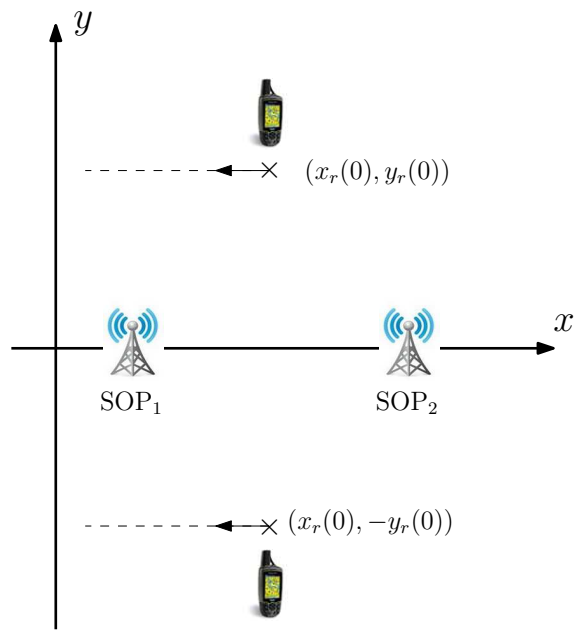

Figure 1. Simple scenario to demonstrate the observability condition with 2 known anchor SOPs

In particular, consider an environment with $\boldsymbol{x}_{r}(0)=[250,250,-1,0]^{\top}, \boldsymbol{x}_{s, a_{1}}(0)=[0,0]^{\top}, \boldsymbol{x}_{s, a_{2}}(0)=$ $[500,0]^{\top}, \tilde{q}_{x}=\tilde{q}_{x}=0.01, \tilde{r}=1$, and $T=1$. Figure 2 shows the estimation error $\tilde{\boldsymbol{x}} \triangleq \boldsymbol{x}-\hat{\boldsymbol{x}}$ along with the $2 \mathbf{P}\left(t_{k} \mid t_{k}\right)$ estimation error covariance bounds achieved after 50 Monte Carlo (MC) simulation runs when the observations where filtered through an EKF with an initial state estimate $\hat{\boldsymbol{x}}(0 \mid-1)=$ $[220,220,-5,5,0,0,500,0]^{\top}$. It is obvious that the estimates indeed converge to the true states and that the estimation errors remain bounded. In contrast, the same simulation was conducted while initializing the initial state estimate at $\hat{\boldsymbol{x}}(0 \mid-1)=[220,-220,-5,5,0,0,500,0]^{\top}$. Figure 3 shows the resulting estimation error $\tilde{\boldsymbol{x}}$ along with the $2 \mathbf{P}\left(t_{k} \mid t_{k}\right)$ estimation error covariance bounds achieved after $50 \mathrm{MC}$ simulation runs. It can be noted that while the estimation error converges and remains bounded, it converges to an incorrect trajectory- the one which would have started from a receiver initial condition at $\boldsymbol{x}_{r}(0)=[250,-250,-1,0]^{\top}$.

Of course, adding a third known SOP resolves this ambiguity. So why are the nonlinear and linear observability analyses revealing that we need only 2 known anchor SOPs? The answer to this question stems from the fact that the nonlinear observability analysis only guarantees weak local observability, namely the existence of a neighborhood within which the initial conditions are distinguishable. For the scenario depicted in Figure 1, such neighborhood turns out to be a half-plane around the initial condition. On the other hand, the fact that we are linearizing the nonlinear observations first implies that our LTV results are valid only locally, i.e. in the neighborhood where the linearizations are valid. Another important conclusion from this analysis is that the observability analysis through the PWCS theory, as applied, is not appropriate for analyzing the OpNav environment. The confusion arising from the conclusions achieved by this theory, demonstrated in this simple example, are similar to the ones encountered in the SLAM observability analysis. ${ }^{6-11}$ The reason behind the encountered discrepancies is that we cannot simply take 
the time segment $j$ to coincide with the discretization instant $t_{k}$, since each time segment should contain $n$ measurement samples, while the Jacobian matrices $\mathbf{F}$ and $\mathbf{H}$ are held constant within such segment. Therefore, in the forthcoming analyses we will only employ the nonlinear observability test and the linear observability test through the $l$-step observability matrix. The reason that both analyses are carried out is that while linear observability is sufficient to establish nonlinear weak local observability, the converse is not necessarily true, i.e. linear observability could conclude that a system is not observable, while nonlinear observability analysis could reveal the contrary.
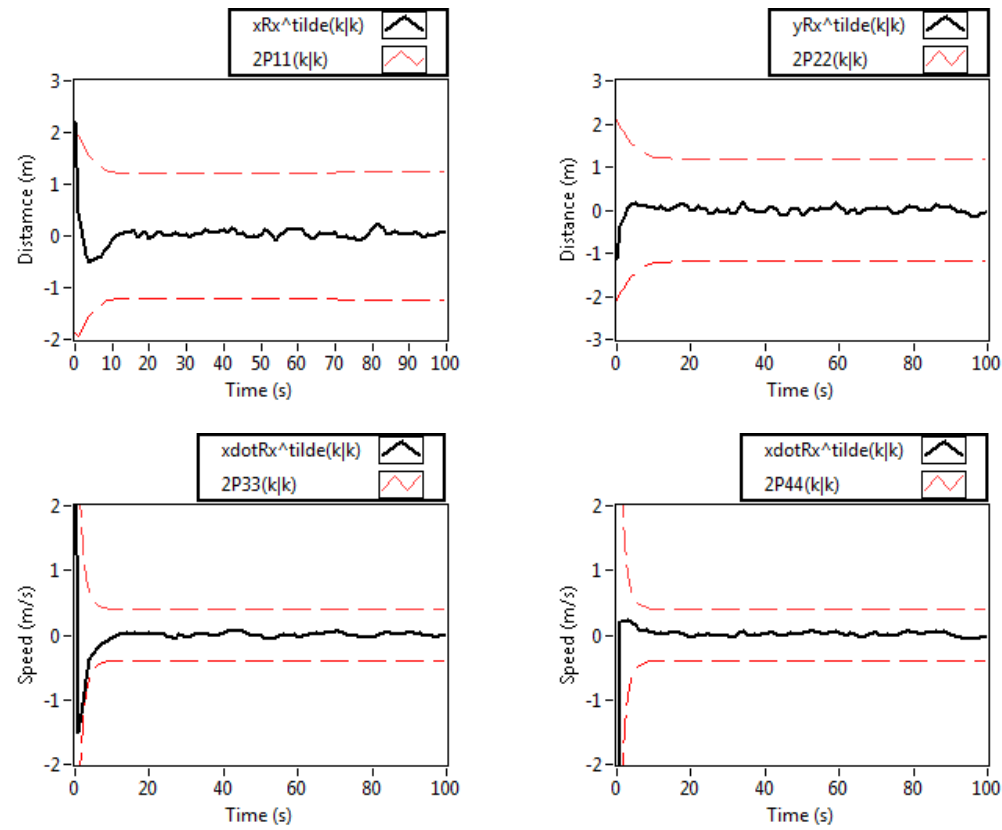

Figure 2. Estimation error trajectories and $2 \mathbf{P}\left(t_{k} \mid t_{k}\right)$ bounds achieved for the simple scenario environment with initial state estimate $\hat{\boldsymbol{x}}(0 \mid-1)=[220,220,-5,5,0,0,500,0]^{\top}$


Figure 3. Estimation error trajectories and $2 \mathbf{P}\left(t_{k} \mid t_{k}\right)$ bounds achieved for the simple scenario environment with initial state estimate $\hat{\boldsymbol{x}}(0 \mid-1)=[220,-220,-5,5,0,0,500,0]^{\top}$ 


\section{B. Opportunistic Navigation Observability Analysis}

In this subsection, various scenarios will be considered with respect to what is known in the OpNav environment. Such scenarios are outlined in Table 1. In Table 1, fully-known means that all the initial states are known. Specifically, a fully-known receiver is one with known $\boldsymbol{x}_{r}(0)$, whereas a fully-known SOP is one with known $\boldsymbol{x}_{s}(0)$. On the other hand, partially-known means that only the initial position is known. Specifically, a partially-known receiver is one with known $\boldsymbol{r}_{r}(0)$, whereas a partially-known SOP is one with known $\boldsymbol{r}_{s}(0)$. The objective of this analysis is twofold: (i) determine whether the system is observable, and (ii) if the system is not completely observable, determine the unobservable directions in the state-space. For all the scenarios considered in Table 1, the conclusions achieved through the nonlinear observability analysis coincided with those achieved through the LTV observability analysis.

Table 1. OpNav observability analysis scenarios considered

\begin{tabular}{|l||l|l|}
\hline Case & Receiver & SOP \\
\hline \hline 1 & Unknown & Unknown \\
\hline 2 & Unknown & Partially-known \\
\hline 3 & Unknown & Fully-known \\
\hline 4 & Fully-known & Unknown \\
\hline 5 & Partially-known & Unknown \\
\hline 6 & Partially-known & Partially-known \\
\hline 7 & Partially-known & Fully-known \\
\hline
\end{tabular}

Case 1. The LTV observability analysis concluded that this scenario is unobservable. The rank of the $l$-step observability matrix keeps increasing as we add more time-steps till we reach $l=5$, at which case $\operatorname{rank}\left[\mathcal{O}_{L}(0, l)\right]=5$ and the rank never improves any further. The resulting null-space of $\mathcal{O}_{L}(0, l)$ turns out to be

$$
\mathcal{N}\left[\mathcal{O}_{L}(0, l)\right]=\operatorname{span}\left[\begin{array}{cccccccccc}
0 & 0 & 0 & 0 & 0 & 1 & 0 & 0 & 0 & 1 \\
0 & 0 & 0 & 0 & 1 & 0 & 0 & 0 & 1 & 0 \\
0 & 1 & 0 & 0 & 0 & 0 & 0 & 1 & 0 & 0 \\
1 & 0 & 0 & 0 & 0 & 0 & 1 & 0 & 0 & 0 \\
\frac{-y_{r}+y_{s}}{\dot{x}_{r}} & \frac{x_{r}-x_{s}}{\dot{x}_{r}} & -\frac{\dot{y}_{r}}{\dot{x}_{r}} & 1 & 0 & 0 & 0 & 0 & 0 & 0
\end{array}\right]^{\top}=\operatorname{span}\left[\begin{array}{lllll}
\mathbf{n}_{1} & \mathbf{n}_{2} & \mathbf{n}_{3} & \mathbf{n}_{4} & \mathbf{n}_{5}
\end{array}\right] .
$$

The structure of $\mathcal{N}\left[\mathcal{O}_{L}(0, l)\right]$ reveals the following conclusions. First, we note that there does not exist a row of zeros throughout the different null-space basis vectors $\left\{\mathbf{n}_{i}\right\}_{i=1}^{5}$. Hence, none of the states is orthogonal to the unobservable subspace and all the states lie within the unobservable subspace. Therefore, none of the states is completely observable (directly). Second, shifting the receiver and SOP positions by $\alpha$ units in the $x$-direction and $\beta$ units in the $y$-direction, where $\alpha, \beta \in \mathbb{R}$, will be unobservable, since this shift, denoted as $\gamma=\alpha \mathbf{n}_{3}+\beta \mathbf{n}_{4}$ lies in the null-space of $\mathcal{O}_{L}(0, l)$. The nonlinear observability analysis reveals the same conclusions in that $\mathcal{O}_{\mathrm{NL}}$ is rank-deficient by 5 . Moreover, the basis for $\mathcal{O}_{\mathrm{NL}}^{\perp}$ turns out to be identical to those of $\mathcal{N}\left[\mathcal{O}_{L}(0, l)\right]$. These results re-affirm our physical intuition that such environment is unobservable. It is worth noting that performing the observability analysis based on the PWCS theory reveals that this system is unobservable, but the receiver's velocity states, $\dot{x}_{r}$ and $\dot{y}_{r}$, are observable. This contradicts with our physical intuition and with the results achieved through the nonlinear and LTV observability analyses.

Case 2. The LTV observability analysis concludes that this scenario is unobservable. The rank of the $l$-step observability matrix keeps increasing as we add more time-steps till we reach $l=5$, at which case $\operatorname{rank}\left[\mathcal{O}_{L}(0, l)\right]=7$ and the rank never improves any further. The resulting null-space of $\mathcal{O}_{L}(0, l)$ turns out to be

$$
\mathcal{N}\left[\mathcal{O}_{L}(0, l)\right]=\operatorname{span}\left[\begin{array}{cccccccccc}
0 & 0 & 0 & 0 & 0 & 1 & 0 & 0 & 0 & 1 \\
0 & 0 & 0 & 0 & 1 & 0 & 0 & 0 & 1 & 0 \\
\frac{-y_{r}+y_{s}}{\dot{x}_{r}} & \frac{x_{r}-x_{s}}{\dot{x}_{r}} & -\frac{\dot{y}_{r}}{\dot{x}_{r}} & 1 & 0 & 0 & 0 & 0 & 0 & 0
\end{array}\right]^{\top}=\operatorname{span}\left[\begin{array}{lll}
\mathbf{n}_{1} & \mathbf{n}_{2} & \mathbf{n}_{3}
\end{array}\right] .
$$


Once again, none of the states is directly observable, except for $x_{s}$ and $y_{s}$, which are observable by construction. The nonlinear observability analysis reveals the same conclusions in that $\mathcal{O}_{\mathrm{NL}}$ is rank-deficient by 3 . Moreover, the basis for $\mathcal{O}_{\mathrm{NL}}^{\perp}$ turns out to be identical to those of $\mathcal{N}\left[\mathcal{O}_{L}(0, l)\right]$.

Case 3. The LTV observability analysis concludes that this scenario is unobservable. The rank of the $l$-step observability matrix keeps increasing as we add more time-steps till we reach $l=4$, at which case $\operatorname{rank}\left[\mathcal{O}_{L}(0, l)\right]=9$ and the rank never improves any further. The resulting null-space of $\mathcal{O}_{L}(0, l)$ turns out to be

$$
\mathcal{N}\left[\mathcal{O}_{L}(0, l)\right]=\operatorname{span}\left[\begin{array}{llllllllll}
\frac{-y_{r}+y_{s}}{\dot{x}_{r}} & \frac{x_{r}-x_{s}}{\dot{x}_{r}} & \frac{\dot{y}_{r}}{\dot{x}_{r}} & 1 & 0 & 0 & 0 & 0 & 0 & 0
\end{array}\right]^{\top}=\operatorname{span}\left[\mathbf{n}_{1}\right] .
$$

The structure of $\mathcal{N}\left[\mathcal{O}_{L}(0, l)\right]$ reveals that the receiver clock bias $\delta t_{r}$ and clock drift $\dot{\delta} t_{r}$ are indeed observable as they are orthogonal to the unobservable subspace, whose dimension is 1 , and is spanned by the vector $\mathbf{n}_{1}$. The nonlinear observability analysis reveals the same conclusions in that $\mathcal{O}_{\mathrm{NL}}$ is rank-deficient by 1. Moreover, the basis for $\mathcal{O}_{\mathrm{N} L}^{\perp}$ turns out to be identical to that of $\mathcal{N}\left[\mathcal{O}_{L}(0, l)\right]$.

Case 4. The LTV observability analysis concludes that this scenario is observable. The $l$-step observability matrix becomes full-rank in $l=4$ steps, rendering the system completely observable. Nonlinear observability analysis concludes that this scenario is indeed locally weakly observable.

Case 5. The LTV observability analysis concludes that this scenario is unobservable. The rank of the $l$-step observability matrix keeps increasing as we add more time-steps till we reach $l=4$, at which case $\operatorname{rank}\left[\mathcal{O}_{L}(0, l)\right]=8$ and the rank never improves any further. The resulting null-space of $\mathcal{O}_{L}(0, l)$ turns out to be

$$
\mathcal{N}\left[\mathcal{O}_{L}(0, l)\right]=\operatorname{span}\left[\begin{array}{llllllllll}
0 & 0 & 0 & 0 & 0 & 1 & 0 & 0 & 0 & 1 \\
0 & 0 & 0 & 0 & 1 & 0 & 0 & 0 & 1 & 0
\end{array}\right]^{\top}=\operatorname{span}\left[\begin{array}{ll}
\mathbf{n}_{1} & \mathbf{n}_{2}
\end{array}\right] .
$$

The structure of the null-space of $\mathcal{O}_{L}(0, l)$ reveals that receiver's velocity states $\dot{x}_{r}$ and $\dot{y}_{r}$ as well as SOP's position states $\left(x_{s}, y_{s}\right)$ are observable, since the elements in their corresponding rows in the basis vectors of the null-space are zeros. Moreover, we note that the system is unobservable with respect to a translation in the $\delta t-\dot{\delta} t$ space of the receiver and SOP. The nonlinear observability analysis reveals the same conclusions in that $\mathcal{O}_{\mathrm{NL}}$ is rank-deficient by 2 . Moreover, the basis for $\mathcal{O}_{\mathrm{NL}}^{\perp}$ turns out to be identical to those of $\mathcal{N}\left[\mathcal{O}_{L}(0, l)\right]$.

Case 6. The LTV observability analysis concludes that this scenario is unobservable. The rank of the $l$-step observability matrix increases in two time-steps to be of rank 8 and never improves beyond that point. The resulting null-space of $\mathcal{O}_{L}(0, l)$ turns out to be

$$
\mathcal{N}\left[\mathcal{O}_{L}(0, l)\right]=\operatorname{span}\left[\begin{array}{llllllllll}
0 & 0 & 0 & 0 & 0 & 1 & 0 & 0 & 0 & 1 \\
0 & 0 & 0 & 0 & 1 & 0 & 0 & 0 & 1 & 0
\end{array}\right]^{\top}=\operatorname{span}\left[\begin{array}{ll}
\mathbf{n}_{1} & \mathbf{n}_{2}
\end{array}\right] .
$$

The structure of the null-space of $\mathcal{O}_{L}(0, l)$ reveals that none of the clock error states are observable and that we cannot observe any translation in the $\delta t-\dot{\delta} t$ space of the receiver and SOP. The nonlinear observability analysis reveals the same conclusions in that $\mathcal{O}_{\mathrm{NL}}$ is rank-deficient by 2. Moreover, the basis for $\mathcal{O}_{\mathrm{NL}}^{\perp}$ turns out to be identical to that of $\mathcal{N}\left[\mathcal{O}_{L}(0, l)\right]$.

Case 7. The LTV observability analysis concludes that this scenario is observable. The rank of the $l$-step observability matrix increases in two time-steps to be of full-rank, deeming the system completely observable. Nonlinear observability analysis concludes that this scenario is indeed locally weakly observable.

In summary, we can generalize this analysis into the following important result.

Result 1 An opportunistic navigation environment with one receiver and $n$ SOPs is completely observable if any of the following conditions is satisfied

- Fully-known receiver's initial state and and n unknown SOPs

- Partially-known receiver's initial state and one fully-known SOP's initial state 


\section{Simulations and Results}

In this section, simulation results that were achieved for the two observable cases in Table 1 are presented. For purposes of numerical stability, the clock error states were defined to be $c \delta t$ and $c \dot{\delta t}$. The simulation for Case 4 considered an environment with a fully-known receiver with an initial state $\boldsymbol{x}_{r}(0)=$ $[100,100,10,-10,0,0]^{\top}$, and 2 unknown SOPs with initial states $\boldsymbol{x}_{s_{1}}(0)=[0,0,10,0]^{\top}$ and $\boldsymbol{x}_{s_{2}}(0)=$ $[200,200,0,0]^{\top}$. The receiver's clock oscillator was assumed to be a temperature-compensated crystal oscillator (TCXO) with $h_{0}=2 \times 10^{-19}$ and $h_{-2}=2 \times 10^{-20}$, while the SOPs' clocks oscillators were assumed to be oven-controlled crystal oscillators (OCXOs) with $h_{0}=8 \times 10^{-20}$ and $h_{-2}=4 \times 10^{-23}$. The process and observation noise spectral densities were taken to be $\tilde{q}_{x}=\tilde{q}_{x}=0.01$ and $\tilde{r}=1$, respectively, and the sampling time was set to $T=1$. The initial state estimate of the EKF was taken to be $\hat{\boldsymbol{x}}(0 \mid-1)=[100,100,10,-10,0,0,10,10,30,3,220,220,2,2]^{\top}$, and the initial covariance of estimation error was taken to be $\mathbf{P}(0 \mid-1)=\operatorname{diag}\left[\mathbf{0}_{6 \times 6}, 100 \mathbf{I}_{4 \times 4}, 100 \mathbf{I}_{4 \times 4}\right]$. Figure 4 shows the resulting estimation error $\tilde{\boldsymbol{x}}$ along with the $2 \mathbf{P}\left(t_{k} \mid t_{k}\right)$ estimation error covariance bounds achieved after $50 \mathrm{MC}$ simulation runs for $\mathrm{SOP}_{1}$ states. Comparable results were noted for $\mathrm{SOP}_{2}$. It is noted that the estimation error covariance converge and that the estimation errors remain bounded.

The simulation for Case 7 considered an environment with a partially-known receiver with an initial state $\boldsymbol{x}_{r}(0)=[100,100,10,-10,0,0]^{\top}$, a fully known SOP with an initial state $\boldsymbol{x}_{s}(0)=[0,0,0,0]^{\top}$, and an unknown SOP with an initial state $\boldsymbol{x}_{s_{2}}(0)=[200,200,0,0]^{\top}$. The process and observation noise spectral densities were taken to be $\tilde{q}_{x}=\tilde{q}_{x}=0.01$ and $\tilde{r}=1$, respectively, and the sampling time was set to $T=1$. The receiver's clock oscillator was assumed to be a TCXO with $h_{0}=2 \times 10^{-19}$ and $h_{-2}=2 \times 10^{-20}$, while the SOPs' clocks oscillators were assumed to be OCXOs with $h_{0}=8 \times 10^{-20}$ and $h_{-2}=4 \times 10^{-23}$. The initial state estimate of the EKF was taken to be $\hat{\boldsymbol{x}}(0 \mid-1)=[100,100,5,-5,3,-3,0,0,0,0,220,220,2,2]^{\top}$, and the initial covariance of estimation error was taken to be $\mathbf{P}(0 \mid-1)=\operatorname{diag}\left[\mathbf{0}_{2 \times 2}, 10 \mathbf{I}_{4 \times 4}, \mathbf{0}_{4 \times 4}, 100 \mathbf{I}_{4 \times 4}\right]$. Figure 5 shows the resulting estimation error $\tilde{\boldsymbol{x}}$ along with the $2 \mathbf{P}\left(t_{k} \mid t_{k}\right)$ estimation error covariance bounds achieved after $50 \mathrm{MC}$ simulation runs for the estimated receiver states. It is noted that the estimation error covariance converge and that the estimation errors remain bounded.
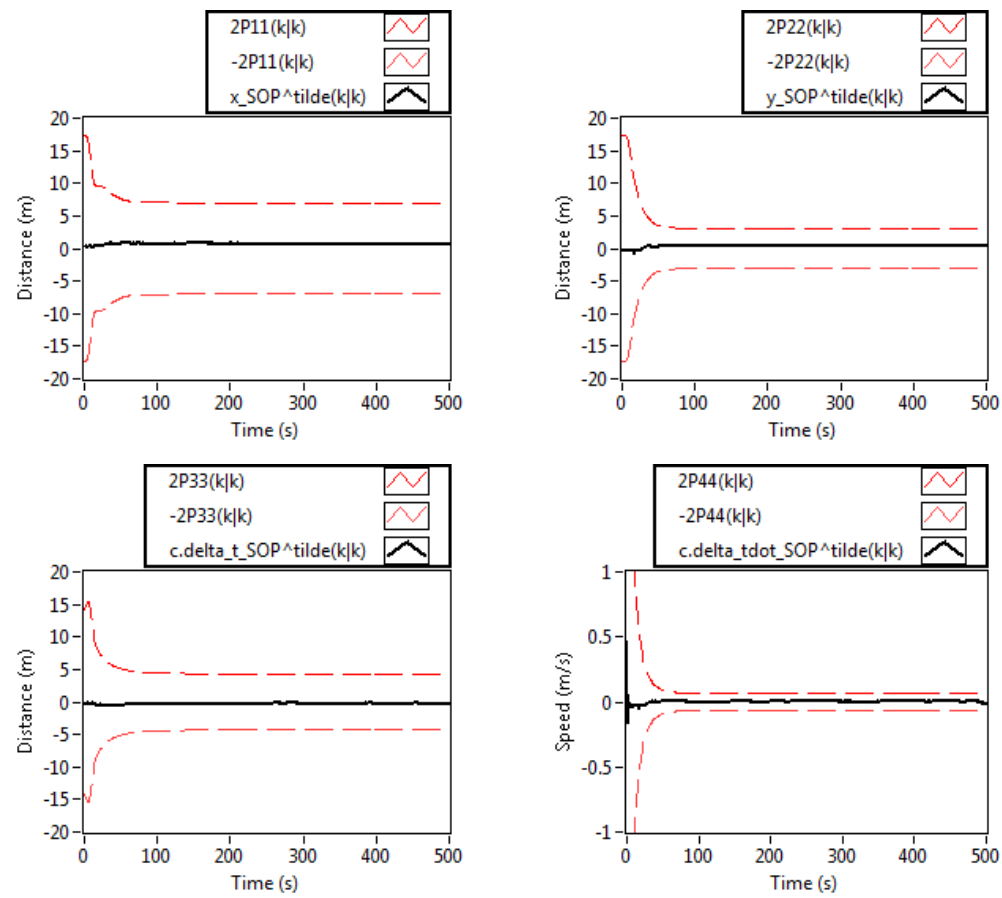

Figure 4. Estimation error trajectories and $2 \mathbf{P}\left(t_{k} \mid t_{k}\right)$ bounds achieved for case 4 in Table 1: a fully-known receiver and unknown SOP 



Figure 5. Estimation error trajectories and $2 \mathbf{P}\left(t_{k} \mid t_{k}\right)$ bounds achieved for simulating case 7 in Table 1: a partially-known receiver and a fully-known SOP

\section{Conclusion}

Analysis of observability, convergence, and estimability properties for OpNav are of paramount importance before deploying OpNav-enabled receivers in the real-world. This paper addressed the first of these three analysis subject matters. To this end, three candidate observability analysis tools, namely nonlinear weak local observability, observability of LTV systems, and observability of PWCSs, were considered. These observability tools were evaluated and it was concluded that observability via PWCS theory is inappropriate to assess OpNav observability. Subsequently, several OpNav scenarios were considered and their respective observability were analyzed. It was concluded that in an OpNav environment of one receiver and $n$ SOPs, the OpNav environment is completely observable if the initial states of a receiver are known or the initial position of the receiver is known along with the initial state of one SOP. Future work will consider theoretical analysis of more complex OpNav scenarios along with corresponding simulations. Moreover, the OpNav environment will be extended to include multiple receivers. Subsequently, observability will be analyzed for various scenarios of such collaborative OpNav environment.

\section{Acknowledgments}

The authors would like to thank Professors Ari Arapostathis and Maruthi Akella. Their feedback and helpful discussions have added value to this research and is much appreciated.

\section{References}

${ }^{1}$ K. Pesyna, Z. Kassas, J. Bhatti, and T. Humphreys, "Tightly-coupled opportunistic navigation for deep urban and indoor positioning," in Proceedings of the International Technical Meeting of The Satellite Division of the Institute of Navigation (ION GNSS), vol. 1, September 2011, pp. 3605-3617.

${ }^{2}$ K. M. Pesyna, Jr., K. Wesson, R. W. Heath, Jr., and T. E. Humphreys, "Extending the reach of GPS-assisted femtocell synchronization and localization through tightly-coupled opportunistic navigation," in IEEE GLOBECOM Workshops, 2011.

${ }^{3} \mathrm{H}$. Durrant-Whyte and T. Bailey, "Simultaneous localization and mapping: part I," IEEE Robotics 8 Automation Magazine, vol. 13, no. 2, pp. 99-110, June 2006.

${ }^{4}$ T. Bailey and H. Durrant-Whyte, "Simultaneous localization and mapping: part II," IEEE Robotics E Automation Magazine, vol. 13, no. 3, pp. 108-117, September 2006. 
${ }^{5}$ J. J. Spilker, Jr, Global Positioning System: Theory and Applications. Washington, D.C.: American Institute of Aeronautics and Astronautics, 1996, ch. 2: Overview of GPS Operation and Design, pp. 57-119.

${ }^{6} \mathrm{~J}$. Andrade-Cetto and A. Sanfeliu, "The effects of partial observability in SLAM," in Proceedings of IEEE Internartional Conference on Robotics and Automation, vol. 1, April 2004, pp. 397-402.

7 _, "The effects of partial observability when building fully correlated maps," IEEE Transactions on Robotics, vol. 21, no. 4, pp. 771-777, August 2005.

${ }^{8}$ T. Vida-Calleja, M. Bryson, S. Sukkarieh, A. Sanfeliu, and J. Andrade-Cetto, "On the observability of bearing-only SLAM," in Proceedings of IEEE Internartional Conference on Robotics and Automation, vol. 1, April 2007, pp. 4114-4118.

${ }^{9}$ K. Lee, W. Wijesoma, and I. Javier, "On the observability and observability analysis of SLAM," in Proceedings of IEEE Internartional Conference on Intelligent Robots and Systems, vol. 1, October 2006, pp. 3569-3574.

${ }^{10} \mathrm{Z}$. Wang and G. Dissanayake, "Observability analysis of SLAM using Fisher information matrix," in Proceedings of IEEE International Conference on Control, Automation, Robotics, and Vision, vol. 1, December 2008, pp. 1242-1247.

${ }^{11}$ L. Perera, A. Melkumyan, and E. Nettleton, "On the linear and nonlinear observability analysis of the SLAM problem," in Proceedings of IEEE Internartional Conference on Mechatronics, vol. 1, April 2009, pp. 1-6.

${ }^{12}$ L. Perera and E. Nettleton, "On the nonlinear observability and the information form of the SLAM problem," in Proceedings of IEEE Internartional Conference on Intelligent Robots and Systems, vol. 1, October 2009, pp. 2061-2068.

${ }^{13} \mathrm{G}$. Huang, A. Mourikis, and S. Roumeliotis, "Analysis and improvement of the consistency of extended Kalman filter nased SLAM," in Proceedings of IEEE Internartional Conference on Robotics and Automation, vol. 1, May 2008, pp. 473-479.

14 __ , "Observability-based rules for designing consistent EKF SLAM estimators," International Journal of Robotics Research, vol. 29, no. 5, pp. 502-528, April 2010.

${ }^{15} \mathrm{D}$. Goshen-Meskin and I. Bar-Itzhack, "Observability analysis of piece-wise constant systems-part I: Theory," IEEE Transactions on Aerospace and Electronic Systems, vol. 28, no. 4, pp. 1056-1067, October 1992.

${ }^{16} \mathrm{M}$. Bryson and S. Sukkarieh, "Observability analysis and active control for airborne SLAM," IEEE Transactions on Aerospace and Electronic Systems, vol. 44, no. 1, pp. 261-280, January 2008

${ }^{17}$ R. Hermann and A. Krener, "Nonlinear controllability and observability," IEEE Transactions on Automatic Control, vol. 22, no. 5, pp. 728-740, October 1977.

${ }^{18}$ W. Rugh, Linear System Theory, 2nd ed. Upper Saddle River, NJ: Prentice Hall, 1996.

${ }^{19} \mathrm{M}$. Anguelova, "Observability and identifiability of nonlinear systems with applications in biology," Ph.D. dissertation, Chalmers University Of Technology and Göteborg University, Sweden, 2007.

${ }^{20} \mathrm{~J}$. Casti, "Recent developments and future perspectives in nonlinear system theory," SIAM Review, vol. 24, no. 3, pp. 301-331, July 1982

${ }^{21}$ W. Respondek, "Geometry of static and dynamic feedback," in Lecture Notes at the Summer School on Mathematical Control Theory, Trieste, Italy, September 2001.

${ }^{22}$ J. Barnes, A. Chi, R. Andrew, L. Cutler, D. Healey, D. Leeson, T. McGunigal, J. Mullen, W. Smith, R. Sydnor, R. Vessot, and G. Winkler, "Characterization of frequency stability," IEEE Transactions on Instrumentation and Measurement, vol. 20, no. 2, pp. 105-120, May 1971.

${ }^{23}$ Y. Bar-Shalom, X. Li, and T. Kirubarajan, Estimation with Applications to Tracking and Navigation, 1st ed. New York, NY: John Wiley \& Sons, 2002.

${ }^{24}$ R. Brown and P. Hwang, Introduction to Random Signals and Applied Kalman Filtering, 3rd ed. John Wiley \& Sons, 2002.

${ }^{25}$ M. Psiaki and S. Mohiuddin, "Modeling, analysis, and simulation of GPS carrier phase for spacecraft relative navigation," Journal of Guidance, Control, and Dynamics, vol. 30, no. 6, pp. 1628-1639, November-December 2007.

${ }^{26}$ Z. Kassas, "Discretisation of continuous-time dynamic multi-input multi-output systems with non-uniform delays," IET Proceedings on Control Theory \& Applications, vol. 5, no. 14, pp. 1637-1647, September 2011. 